\title{
Cognitive and Behavioral Changes After Surgery and Deep Brain Stimulation in Parkinson's Disease
}

\author{
Mervat Wahba* \\ Department of Neurology, The University of Tennessee Health Science Center, USA
}

*Corresponding author: Mervat Wahba, Department of Neurology, The University of Tennessee Health Science Center, USA.
Received Date: June 03, 2019

Published Date: June 11, 2019

\section{Introduction}

Surgical interventions for Parkinson's disease may be a treatment option in predominantly unilateral symptoms in patients whose symptoms are not adequately managed by other treatment modalities. Eligibility of the patients who are good surgical candidates must be carefully analyzed. General surgical complications as well as post-operative cognitive and mood changes must be assessed. Idiopathic Parkinson disease is a Neuro degenerative disease that is characterized by resting tremor, bradykinesia, rigidity and in later postural instability. The loss of dopaminergic cells in the substantia nigra pars compacta accounts for the motor dysfunction in Parkinson disease. In Parkinson disease there is disruption of normal excitatory and inhibitory influences within the basal ganglia most pronounced in the ventrolateral part of the substantia nigra that innervates the posterior putamen, this causes abnormal activity in the thalamocortical circuits. In the direct pathway, the stratum receives excitatory input from the cortical regions and projects inhibitory efferent to the GPI/SNr complex. These structures have an inhibitory effect on the thalamus which subsequently projects excitatory efferent to the cortex. The indirect pathway exerts inhibitory effect that projects from the striatum to the Globus pallidus externa then to the STN which then projects excitatory effects to the GPI-SN-pr complex (Figure 1). In the post-levodopa era, several neuroanatomical targets such as the thalamus, GPI and STN can be approached with surgical techniques as ablation stimulation and transplantation to alleviate the motor symptoms associated with PD.
A

A

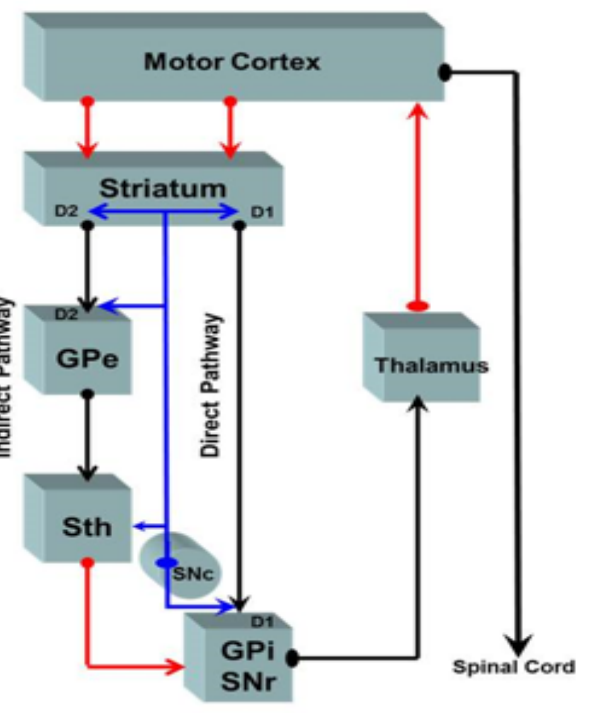

B

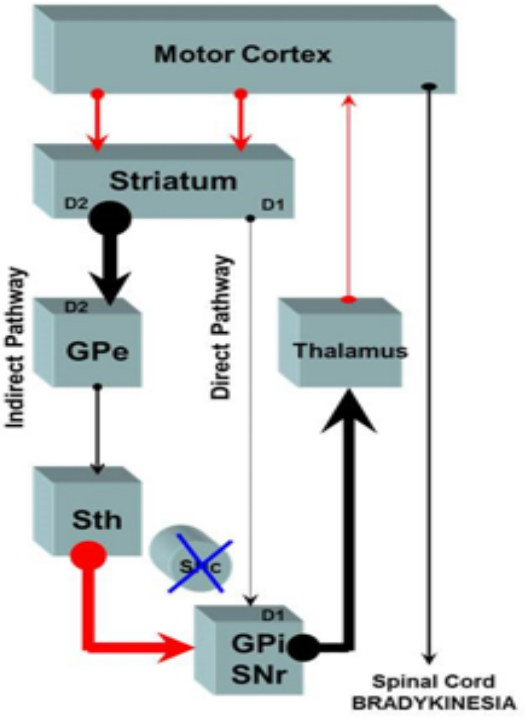

C

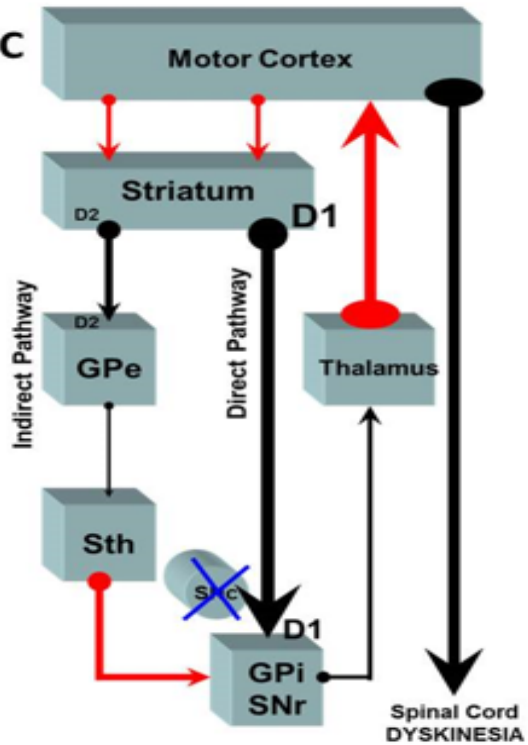

Figure 1: Reference Open access peer-reviewed chapter. Pathophysiology of L-Dopa Induced Dyskinesia-Changes in D1/D3 Receptors and Their Signaling Pathway. 


\section{Neuro Behavioral Outcomes After Surgical} Lesioning of the Ventrolateral and Ventral Anterior Nuclei of the Thalamus

The cognitive morbidity after ventrolateral and ventral interior thalamus surgery cannot be ascertained because of the

different methods of assessment measures, and different surgical approaches.

Modern thalamotomy may be safer than the earlier operations. Gamma knife thalamotomy was reported to have a complication rate of 1.5 to $2 \%$ [1]. In a case series of unilateral thalamotomy (28 right and 25 left), formal neuropsychological measures showed little change three months after surgery [2].

Burchiel KJ [3] noted that 39\% of thalamotomy patients demonstrated decline in speech, language and memory which was more prevalent among bilateral then unilateral operates $60 \%$ versus 31\% Case series and studies found that verbal memory declined after unilateral left and bilateral thalamotomy Shapiro DY, et al. [4] but not after unilateral right thalamotomy [5]. Transient attention declines Riklan M, et al. [6], transient reduction in verbal fluency were also found after left and bilateral thalamotomy.

\section{Postoperative Changes in Mood and Behavior}

A study by Angelini and colleagues [7] reported cases of depression following surgery. Several investigators in contrast have reported improvement in depressive and obsessive symptomatology postoperatively [8]. Cases of bilateral thalamotomy are more likely to display persistent neuropsychiatric changes.

\section{Ventral Intermediate Nucleus Deep Brain Stimulation, Postoperative the Changes in Cognition}

Thalamic stimulation does not appear to cause decline in verbal fluency or memory. Improvements on tasks of problemsolving, verbal fluency, naming and delayed recall were observed up to 12 months [9]. Stimulation parameters may also play a role in cognitive findings. Studies employing intraoperative lower frequencies $60 \mathrm{~Hz}$ were more predictive of memory impairment than higher frequencies $200 \mathrm{~Hz}$, the latter are employed in DBS. No significant depression was detected at three months following surgery in patients who demonstrated improvement in motor functioning [10].

\section{Globus Pallidus Surgical Lesioning Gpi}

In a meta-analysis by Alkhani and Lozano it was reported that transient memory deficits occur in $1.3 \%$ of cases after unilateral or bilateral pallidotomy, persistent memory deficits of good in less than $1 \%$ of cases [11]. The most frequently reported postoperative cognitive change is decline in the verbal fluency after left pallidotomy, these are not evident after unilateral right pallidotomy. Depressive symptoms were reported in some cases after that globus pallidotomy however several studies have reported no significant change in mood state [12].

\section{Deep Brain Stimulation GPI}

It was reported that decline in visual construction ability and verbal fluency of non-significant clinical importance have occurred [13].

\section{Changes in Mood and Behavior}

Unilateral GPI deep brain stimulation did not induce depressive symptoms. One study reported as single patient who underwent bilateral staged GPI DBS surgery and developed manic episodes after deep brain surgery both with unilateral and bilateral stimulation. This improved after reduction in his Levodopa dosage [14].

\section{Subthalamic Nucleus Surgical Lesioning}

Sub thalamotomy is avoided for concern of causing hemiballismus. Spiegel E, et al [15]. report a $30 \%$ incidence of psycho-organic syndrome out of 33 patients who underwent sub thalamic ablation surgery.

\section{Deep Brain Supplement Simulation}

Cognitive outcomes after bilateral STN-DBS varies across studies. Ardouin C, et al [16] reported changes in cognition 3 to 6 months after bilateral subthalamic DBS. Cognitive decline was observed on verbal fluency tasks

\section{Changes in Mood and Behavior}

Several studies reported improvement in depression [16]. Signs of perseveration, impulsivity, diminished social judgment have been reported [17].

\section{Summary}

Surgical interventions for Parkinson's disease may be relatively safe from the cognitive standpoint. Decline in verbal fluency was noted after left-sided surgeries. Isolated cases of psychiatric decline have been reported, more noted in patients who are elderly and with a baseline of dementia. Formal assessment with standardized neuropsychological testing is needed to determine the possible side effects of surgery or DBS. DBS appears to be safer than ablation surgery.

\section{Acknowledgement}

None.

\section{Conflict of interest}

No conflict of interest.

\section{References}

1. Young RF (2001) Gamma knife treatment for movement disorders. Semin Neurosurg 12: 233-243.

2. Lund Johansen M, Hugdahl K, Wester K (1996) Cognitive functions in patients with Parkinson Disease undergoing stereotaxic thalamotomy. J Neurol Neurosurg Psychiatry 60(5): 564-571.

3. Burchiel KJ (1995) Thalamotomy for movement disorders. Neurosurg Clin North Am 6(1): 55-71.

4. Shapiro DY, Sadowsky DA, Henderson WG, Van Buren JM (1973) An assessment of cognitive function in postthalamotomy Parkinson patients. Confin Neurolo 35(3): 144-166. 
5. Riklan M, Diller L, Weiner H, Cooper IS (1960) Psychological studies on effects of chemosurgery of the basal ganglia in Parkinsonism; Intellectual functioning. Arch Gen Psychiatry 2: 22-31.

6. Riklan M, Levita E (1970) Psychological studies of thalamic lesions in humans. J Nerv Ment Dis 150(4): 251-265.

7. Angelini L, Nardocci N, Bobo R, Broggi G (1982) Depression after stereotactic thalamotomy in patients with abnormal movements. Ital J Neurol Sci 3(4): 301-310.

8. Hays P, Kirkler B, Walsh LS, Woolfson G (1966) Psychological changes following surgical treatment of Parkinsonsim. Am J Psychiatry 123(6): 657-663.

9. Caparros Lefebvre, Blond S, Pecheux N, et al. (1992) Evaluation neuropsychologique avant et apres stimulation thalamique chez 9 parkinsoniens. Rev Neurol 148: 117-122.

10. Straits Troster K, Fields JA, Wilkinson SB, Pahwa R, Lyons KE, et al. (2000) Health-related quality of life in Parkinson's disease after pallidotomy and deep brain stimulation. Brain Cogn 42(3): 399-416.

11. Alkhani A, Lozano AM (2001) Pallidotomy for Parkinson disease: a review of contemporary literature. J neurosurg 94(1): 43-49.
12. Uitti RJ, Wharen RE, Duffy JR, Lucas JA, Schneider SL, et al. (2000) Unilateral pallidotomy for Parkinson's disease: speech, motor, and neuropsychological outcome measurements. Parkinsonism Relat Disord 6(3): 133-143.

13. Vingerhoets G, Van Der Linden C, Lannoo E, Vandewalle V, Caemaert J, et al. (1999) Cognitive outcome after unilateral pallidal stimulation in Parkinson's disease. J Neurol Neurosurg Psychiatry 66(3): 297-304.

14. Miyawaki E, Perlmutter JS, Troster AI, Videen TO, Koller WC (2000) The behavioral complications of pallidal stimulation: a case report. Brain Cogn 42(3): 417-434.

15. Spiegel E, Wycus H, Orchinik C, Freed H (1955) Thalamic chronotaraxis. Arch Neurol Psychiatry 73: 469-471.

16. Ardouin C, Pillon B, Peiffer E, Bejjani P, Limousin P, et al. (1999) Bilateral subthalamic or pallidal stimulation for Parkinson's disease affects neither memory nor executive functions: a consecutive series of 62 patients. Ann Neurol 46(2): 217-223.

17. Ronald Pfeiffer, Ivan Bodis-Wollner (2005) Parkinson's Disease and Nonmotor dysfunction. In: Ronald Pfeiffer, Ivan Bodis-Wollner (Eds.), Humana Press Editors. 